\title{
Termination Analysis of Tabled Logic Programs using Mode and Type Information
}

\author{
Sofie Verbaeten ${ }^{\star}$ and Danny De Schreye ${ }^{\star \star}$ \\ Department of Computer Science, K.U.Leuven, Belgium \\ \{sofie, dannyd Ocs.kuleuven.ac.be
}

\begin{abstract}
Tabled logic programming is receiving increasing attention in the Logic Programming community. It avoids many of the shortcomings of SLD(NF) execution and provides a more flexible and efficient execution mechanism for logic programs. In particular, tabled execution of logic programs terminates more often than execution based on SLDresolution. One of the few approaches studying termination of tabled logic programs was developed by Decorte $\boldsymbol{e t}$ al. They present necessary and sufficient conditions for two notions of universal termination under SLG-resolution, the resolution principle of tabling: quasi-termination and (the stronger notion of) LG-termination. Starting from these necessary and sufficient conditions, we introduce sufficient conditions which are stated fully at the clause level and are easy to automatize. To this end, we use mode and type information: we consider simply moded, well-typed programs and queries. We point out how our termination conditions can be automatized, by extending the recently developed constraint-based automatic termination analysis for SLD-resolution by Decorte and De Schreye.
\end{abstract}

\section{Introduction}

Tabled logic programming [5,14], extending standard SLD-resolution with a tabling mechanism, avoids many of the shortcomings of SLD execution and provides a more flexible and often considerably more efficient execution mechanism for logic programs. In particular, tabled execution of logic programs terminates more often than execution based on SLD. So, if a program can be proven to terminate under SLD-resolution (by one of the existing automated techniques surveyed in [6]), then the program will trivially also terminate under SLG-resolution, the resolution principle of tabling [5]. However, since there are programs and queries which terminate under SLG-resolution and not under SLD-resolution, more effective proof techniques can be found. One of the few works studying termination of tabled logic programs is [7], in which necessary and sufficient conditions are given for two notions of universal termination under LG-resolution, i.e. SLGresolution with left-to-right selection rule: namely quasi-termination and (the stronger notion of) LG-termination.

\footnotetext{
* Research Assistant of the Fund for Scientific Research - Flanders (Belgium)(F.W.O.).
}

* Senior Research Associate of F.W.O. - Flanders (Belgium). 
This work is based on [7]. Starting from the necessary and sufficient conditions of [7], we present sufficient conditions for quasi-termination and LGtermination which are stated fully at the clause level. To this end we use mode and type information: we consider simply moded, well-typed programs and queries. Our termination conditions are easy to automatize. In particular, we show how the framework of [8], where a constraint-based automatic termination analysis for LD-resolution (SLD-resolution with left-to-right selection rule) is given, needs to be modified in order to prove quasi-termination and LGtermination in an automatic way.

In the following Section 2 of preliminaries, we first recall the notion of SLGresolution [5] for definite programs. Next, we define the notions of simply modedness, well-typedness and input safe atoms and we give some definitions and properties of norms and level mappings. In the following Section 3, the notion of quasi-termination and its characterisation of [7], namely quasi-acceptability, are introduced. We present an easy to automatize, sufficient condition for quasitermination of simply moded well-typed programs and queries in Subsection 3.1. We point out in Subsection 3.2 how this condition can be automatized, by extending the automatic, constraint-based termination analysis for LD-resolution of [8]. By the lack of space, we do not consider the stronger notion of LGtermination in this paper. Instead, we refer to the full version of the paper, [15], where we present an optimatization of the necessary and sufficient condition of [7] for LG-termination, together with an easy to automatize condition for LGtermination of simply moded well-typed programs and queries. We want to note however that this automatizable condition for LG-termination is obtained from its characterisation in a similar way as the one for quasi-termination. In Section 4 we conclude this paper with a discussion on related works. We refer to the full version of the paper, [15], for more examples and all the proofs.

\section{Preliminaries}

We refer to [12] for the basic concepts of logic programming. Throughout the paper, $P$ will denote a definite logic program. The extended Herbrand Universe, $U_{P}^{E}$, and the extended Herbrand Base, $B_{P}^{E}$, associated with a program $P$ are defined as follows. Let $\operatorname{Term}_{P}$ and $\operatorname{Atom}_{P}$ denote the set of respectively all terms and atoms that can be constructed from the alphabet underlying $P$. The variant relation, denoted $\approx$, defines an equivalence. $U_{P}^{E}$ and $B_{P}^{E}$ are respectively the quotient sets $\operatorname{Term}_{P} / \approx$ and $A \operatorname{tom} m_{P} / \approx$. For any term $t$ (or atom $A$ ), we denote its class in $U_{P}^{E}\left(B_{P}^{E}\right)$ as $\tilde{t}(\tilde{A})$. However, when no confusion is possible, we omit the tildes. We use the abbreviation mgu for most general unifier.

We will refer to SLD-derivations following the left-to-right selection rule as LD-derivations. Other concepts will adopt this naming accordingly. Given $S \subseteq B_{P}^{E}$, by $\operatorname{Call}(P, S)$ we denote the subset of $B_{P}^{E}$ such that $\tilde{B} \in \operatorname{Call}(P, S)$ whenever an element of $\tilde{B}$ is a selected literal in an LD-derivation for some $P \cup\{\leftarrow A\}$, with $\tilde{A} \in S$. Throughout the paper we assume that in any derivation 
of a query w.r.t. a program, representatives of equivalence classes are systematically provided with fresh variables, to avoid the necessity of renaming apart.

\subsection{SLG-Resolution for Definite Programs}

The ideas underlying tabling are very simple. Essentially, under a tabled execution mechanism, answers for selected atoms are stored in a table. When a variant of such an atom is recursively called, the selected atom is not resolved against program clauses, instead, all corresponding answers computed so far are looked up in the table and the corresponding answer substitutions are applied to the atom. This process is repeated for all subsequent computed answer substitutions that correspond to the atom.

We present a non-constructive definition of SLG-resolution, the resolution principle underlying tabling, and refer to $[5,14]$ for more constructive formulations of (variants) of tabled resolution.

Definition 1 (pseudo SLG-tree, pseudo LG-tree). Let $P$ be a definite program, $\mathcal{R}$ a selection rule and $A$ an atom. A pseudo SLG-tree for $P \cup\{\leftarrow A\}$ under $\mathcal{R}$ is a tree $\tau_{A}$ such that:

1. the nodes of $\tau_{A}$ are labeled with goals along with an indication of the selected atom according to $\mathcal{R}$,

2. the root of $\tau_{A}$ is $\leftarrow A$,

3. the children of the root $\leftarrow A$ are obtained by resolution against all matching program clauses in $P$, the arcs are labeled with the corresponding mgu used in the resolution step,

4. the (possibly infinitely many) children of non-root nodes can only be obtained by resolving the selected (using $\mathcal{R}$ ) atom $B$ of the node with clauses of the form $B \theta \leftarrow($ not necessarily in $P)$, the arcs are labeled with the corresponding mgu used in the resolution step (i.e. $\theta$ ).

If $\mathcal{R}$ is the leftmost selection rule, $\tau_{A}$ is called a pseudo LG-tree for $P \cup\{\leftarrow A\}$. We say that a pseudo SLG-tree $\tau_{A}$ for $P \cup\{\leftarrow A\}$ is smaller than another pseudo SLG-tree $\tau_{A}^{\prime}$ for $P \cup\{\leftarrow A\}$ iff $\tau_{A}^{\prime}$ can be obtained from $\tau_{A}$ by attaching new sub-branches to nodes in $\tau_{A}$

$A$ (computed) answer clause of a pseudo $S L G$-tree $\tau_{A}$ for $P \cup\{\leftarrow A\}$ is a clause of the form $A \theta \leftarrow$ where $\theta$ is the composition of the substitutions found on $a$ branch of $\tau_{A}$ whose leaf is labeled by the empty goal.

Intuitively, a pseudo SLG-tree (in an SLG-forest, see Definition 2 below) represents the tabled computation of all answers for a given subgoal labeling the root node of the tree. The trees in the above definition are called pseudo SLG-trees because there is no condition yet on which clauses $B \theta \leftarrow$ exactly are to be used for resolution in point 4 . These clauses represent the answers found (possibly in another tree of the forest) for the selected atom. This interaction between the trees in an SLG-forest is captured in the following definition. 
Definition 2 (SLG-forest, LG-forest). Let $P$ be a definite program, $\mathcal{R}$ be a selection rule and $T$ be a (possibly infinite) set of atoms such that no two different atoms in $T$ are variants of each other. $\mathcal{F}$ is an SLG-forest for $P$ and $T$ under $\mathcal{R}$ iff $\mathcal{F}$ is a set of minimal pseudo SLG-trees $\left\{\tau_{A} \mid A \in T\right\}$ where

1. $\tau_{A}$ is a pseudo SLG-tree for $P \cup\{\leftarrow A\}$ under $\mathcal{R}$,

2. every selected atom $B$ of each node in some $\tau_{A} \in \mathcal{F}$ is a variant of an element $B^{\prime}$ of $T$, such that every clause resolved with $B$ is a variant of an answer clause of $\tau_{B^{\prime}}$ and vice versa, for every answer clause of $\tau_{B^{\prime}}$ there is a variant of this answer clause which is resolved with $B$.

Let $S$ be a set of atoms. An SLG-forest for $P$ and $S$ under $\mathcal{R}$ is an $S L G$-forest for a minimal set $T$ with $S \subseteq T$. If $S=\{A\}$, then we also talk about the $S L G$-forest for $P \cup\{\leftarrow A\}$. An LG-forest is an SLG-forest containing only pseudo LG-trees.

Point 2 of Definition 2, together with the imposed minimality of trees in a forest, now uniquely determines these trees. So we can drop the designation "pseudo" and refer to (S)LG-trees in an (S)LG-forest.

Example 1. Let NAT be the following program defining the natural numbers:

$$
\begin{cases}\operatorname{nat}(0) & \leftarrow \\ \operatorname{nat}(s(X)) & \leftarrow \operatorname{nat}(X)\end{cases}
$$

Let $S=\{\operatorname{nat}(X)\}$. Then the (unique) (S)LG-forest for $P$ and $S$, shown in Fig. 1, consists of a single (S)LG-tree. Note that this tree is infinitely branching.

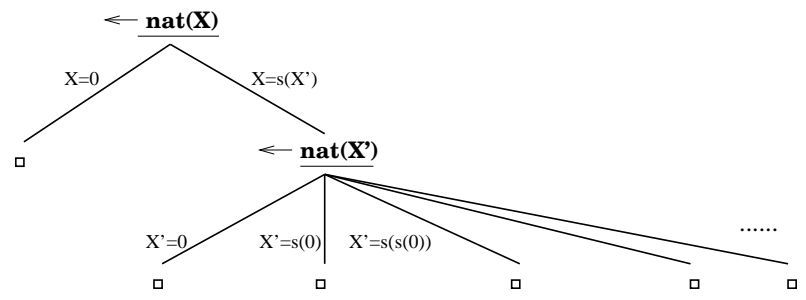

Fig. 1. The SLG-forest for $N A T \cup\{\leftarrow \operatorname{nat}(X)\}$.

We want to note that we can use the notions of LD-derivation and LDcomputation (as they appear in the definition of the call set $\operatorname{Call}(P, S)$ ) even in the context of SLG-resolution, as the set of call patterns and the set of computed answer substitutions are not influenced by tabling; see e.g. [11, Theorem 2.1].

\subsection{Simply Modedness, Well-Typedness and Input Safe Atoms}

Definition 3 (mode for a predicate). Let $p$ be an $n$-ary predicate symbol. $A$ mode for $p$ is a function $m_{p}:\{1, \ldots, n\} \rightarrow\{$ In, Out $\}$. If $m_{p}(i)=$ In (resp. Out), then we say that $i$ is an input (resp. output) position of p (w.r.t. $m_{p}$ ). 
We assume that each predicate symbol has a unique mode. Multiple modes can be obtained by simply renaming the predicates. To simplify the notation, when writing an atom as $p(\mathbf{u}, \mathbf{v})$ we assume that $\mathbf{u}$ is the sequence of terms filling in the input positions of $p$ and $\mathbf{v}$ is the sequence of terms filling in the output positions of $p$. For a term $t$, we denote by $\operatorname{Var}(t)$ the set of variables occurring in $t$. Similar notation is used for sequences of terms.

We introduce the notion of simply modedness [2]. A family of terms is called linear if every variable occurs at most once in it.

Definition 4 (simply modedness). A clause $p_{0}\left(\mathbf{s}_{\mathbf{0}}, \mathbf{t}_{\mathbf{n}+\mathbf{1}}\right) \leftarrow p_{1}\left(\mathbf{s}_{\mathbf{1}}, \mathbf{t}_{\mathbf{1}}\right), \ldots$, $p_{n}\left(\mathbf{s}_{\mathbf{n}}, \mathbf{t}_{\mathbf{n}}\right)$ is called simply moded iff $\mathbf{t}_{\mathbf{1}}, \ldots, \mathbf{t}_{\mathbf{n}}$ is a linear family of variables and for $i \in[1, n]: \operatorname{Var}\left(\mathbf{t}_{\mathbf{i}}\right) \cap\left(\cup_{j=0}^{i} \operatorname{Var}\left(\mathbf{s}_{\mathbf{j}}\right)\right)=\emptyset$.

A program is called simply moded iff every clause of it is simply moded.

A query $\leftarrow p_{1}\left(\mathbf{s}_{\mathbf{1}}, \mathbf{t}_{\mathbf{1}}\right), \ldots, p_{n}\left(\mathbf{s}_{\mathbf{n}}, \mathbf{t}_{\mathbf{n}}\right)$ is simply moded if the clause $p \leftarrow p_{1}\left(\mathbf{s}_{\mathbf{1}}, \mathbf{t}_{\mathbf{1}}\right)$, $\ldots, p_{n}\left(\mathbf{s}_{\mathbf{n}}, \mathbf{t}_{\mathbf{n}}\right)$ is simply moded, where $p$ is any nullary predicate.

For instance, with moding $q($ In, Out $)$, the clause $q(f(X), f(Y)) \leftarrow q(X, Y)$ is simply moded, but the clause $q(X, Y) \leftarrow q(X, f(Y))$ is not. The notion of simply modedness is persistent [2], that is: An LD-resolvent of a simply moded query and a simply moded clause that is variable-disjoint with it, is simply moded. An atom is called input/output disjoint if the family of terms occurring in its input positions has no variable in common with the family of terms occurring in its output positions. As a corollary to the persistence of the notion of simply modedness, we have that: For a simply moded program $P$ and a simply moded query $Q$, all atoms selected in an $L D$-derivation of $P \cup\{\leftarrow Q\}$ are input/output disjoint and such that each of the output positions is filled in by a distinct variable. In [2], it is argued that most programs are simply moded, and that often non-simply moded programs can be naturally transformed into simply moded ones.

We next introduce the notion of input-correct atom w.r.t. a set of atoms. This notion will be useful in our termination condition.

Definition 5 (input-correct atom w.r.t. $S$ ). Let $S \subseteq B_{P}^{E}$ and $A=p(\mathbf{u}, \mathbf{v})$ an atom. We call $A$ input-correct w.r.t. $S$, denoted by $A^{I n} \in S^{I n}$, iff there is an atom $B=p(\mathbf{u}, \mathbf{w})$ such that $\tilde{B} \in S$.

In the sequel, we also use types. A type is defined as a decidable set of terms closed under substitution. A type $T$ is called ground if all its elements are ground, and non-ground otherwise. A typed term is a construct of the form $s: S$, where $s$ is a term and $S$ is a type. Given a sequence $\mathbf{s}: \mathbf{S}=s_{1}: S_{1}, \ldots, s_{n}: S_{n}$ of typed terms, we write $\mathbf{s} \in \mathbf{S}$ iff for every $i \in[1, n]$, we have $s_{i} \in S_{i}$.

Some examples of types are: $U$, the set of all terms; Ground, the set of all ground terms; List, the set of all (possibly non-ground) nil-terminated lists (built on the empty list [ ] and the list constructor [.|.]); and NAT, the set of all natural numbers $\{0, s(0), s(s(0)), \ldots\}$. Throughout the paper, we fix a specific set of types, denoted by Types. We also associate types with predicates.

Definition 6 (type for a predicate, correctly typed). Let $p$ be an $n$-ary predicate symbol. A type for $p$ is a function $t_{p}:\{1, \ldots, n\} \rightarrow$ Types. If $t_{p}(i)=T$, 
we call $T$ the type associated with position $i$ of $p$.

Assuming a type $t_{p}$ for the predicate $p$, we say that an atom $p\left(s_{1}, \ldots, s_{n}\right)$ is correctly typed in position $i$ iff $s_{i} \in t_{p}(i)$. We say that $p\left(s_{1}, \ldots, s_{n}\right)$ is correctly typed iff it is correctly typed in all its positions.

When every considered predicate has a mode and a type associated with it, we can talk about types of input positions and of output positions of an atom. An $n$-ary predicate $p$ with a mode $m_{p}$ and type $t_{p}$ will be denoted by $p\left(<m_{p}(1): t_{p}(1)>, \ldots,<m_{p}(n): t_{p}(n)>\right)$. To simplify the notation, when writing an atom as $p(\mathbf{u}: \mathbf{S}, \mathbf{v}: \mathbf{T})$ we assume that $\mathbf{u}: \mathbf{S}$ is a sequence of typed terms filling in the input positions of $p$ and $\mathbf{v}: \mathbf{T}$ is a sequence of typed terms filling in the output positions of $p$. We call $p(\mathbf{u}: \mathbf{S}, \mathbf{v}: \mathbf{T})$ a typed atom.

Next, we introduce the notion of well-typedness [4]. First, we need the following concept of a type judgement.

Definition 7 ((true) type judgement). A statement of the form $\mathbf{s}: \mathbf{S} \Rightarrow$ $\mathbf{t}: \mathbf{T}$ is called a type judgement. A type judgement $\mathbf{s}: \mathbf{S} \Rightarrow \mathbf{t}: \mathbf{T}$ is true, notated $\models \mathbf{s}: \mathbf{S} \Rightarrow \mathbf{t}: \mathbf{T}$, iff for all substitutions $\theta, \mathbf{s} \theta \in \mathbf{S}$ implies $\mathbf{t} \theta \in \mathbf{T}$.

\section{Definition 8 (well-typedness).}

A clause $p_{0}\left(\mathbf{o}_{\mathbf{0}}: \mathbf{O}_{\mathbf{0}}, \mathbf{i}_{\mathbf{n}+\mathbf{1}}: \mathbf{I}_{\mathbf{n}+\mathbf{1}}\right) \leftarrow p_{1}\left(\mathbf{i}_{\mathbf{1}}: \mathbf{I}_{\mathbf{1}}, \mathbf{o}_{\mathbf{1}}: \mathbf{O}_{\mathbf{1}}\right), \ldots, p_{n}\left(\mathbf{i}_{\mathbf{n}}: \mathbf{I}_{\mathbf{n}}, \mathbf{O}_{\mathbf{n}}: \mathbf{O}_{\mathbf{n}}\right)$ is called well-typed iff for $j \in[1, n+1]: \models \mathbf{O}_{\mathbf{0}}: \mathbf{O}_{\mathbf{0}}, \ldots, \mathbf{O}_{\mathbf{j}-\mathbf{1}}: \mathbf{O}_{\mathbf{j}-\mathbf{1}} \Rightarrow \mathbf{i}_{\mathbf{j}}: \mathbf{I}_{\mathbf{j}}$.

A program is called well-typed iff every clause of it is well-typed. A query $\leftarrow p_{1}\left(\mathbf{i}_{\mathbf{1}}: \mathbf{I}_{\mathbf{1}}, \mathbf{o}_{\mathbf{1}}: \mathbf{O}_{\mathbf{1}}\right), \ldots, p_{n}\left(\mathbf{i}_{\mathbf{n}}: \mathbf{I}_{\mathbf{n}}, \mathbf{O}_{\mathbf{n}}: \mathbf{O}_{\mathbf{n}}\right)$ is well-typed if the clause $p \leftarrow p_{1}\left(\mathbf{i}_{\mathbf{1}}: \mathbf{I}_{\mathbf{1}}, \mathbf{o}_{\mathbf{1}}: \mathbf{O}_{\mathbf{1}}\right), \ldots, p_{n}\left(\mathbf{i}_{\mathbf{n}}: \mathbf{I}_{\mathbf{n}}, \mathbf{o}_{\mathbf{n}}: \mathbf{O}_{\mathbf{n}}\right)$ is well-typed, where $p$ is any nullary predicate.

Note that in a well-typed query, the left-most atom is correctly typed in its input positions. We want to note that the notion of well-modedness [1], is a special case of the notion of well-typedness. Namely, the definition of wellmodedness is an instance of Definition 8, where the types of all positions are (implicitly) defined as Ground (see [1, Theorem 3.7] for more details). Hence, the results in this paper obtained for well-typed programs and queries, also hold for well-moded programs and queries.

As for simply modedness, the notion of well-typedness is persistent [4], that is: An LD-resolvent of a well-typed query and a well-typed clause that is variabledisjoint with it, is well-typed. Hence, we have that: For a well-typed program $P$ and a well-typed query $Q$, all atoms selected in an LD-derivation of $P \cup\{\leftarrow Q\}$ are correctly typed in their input positions.

In [2] the notion of generic expression for a type was introduced. Intuitively, a term $t$ is a generic expression for a type $T$ if it is more general than all elements of $T$ which unify with $t$. This notion is the main tool in the approach of [2] towards replacing unification by iterated matching. It turns out that surprisingly often the input positions of the heads of program clauses are filled in by generic expressions for appropriate types (see [2]). We recall the definition.

Definition 9 (generic expression for a type). Let $T$ be a type. A term $t$ is a generic expression for $T$ iff for every $s \in T$ variable-disjoint with $t$, if $s$ unifies with $t$ then $s$ is an instance of $t$. 
For example, []$,[X],[X \mid Y],[X, Y \mid Z], \ldots$ are generic expressions for the type List; $0, s(X), s(s(s(X))), \ldots$ are generic expressions for the type NAT. A term of the form $f\left(X_{1}, \ldots, X_{m}\right)$ with $X_{1}, \ldots, X_{m}$ a sequence of different variables is called a pure variable term. In [2, Lemma 3.7], it was shown that: variables are generic expressions for any type; the only generic expressions for type $U$ are variables; if the type $T$ does not contain variables, then every pure variable term is a generic expression for $T$; and if $T$ is Ground, then every term is a generic expression for $T$. In [2], the notion of input safe atom was introduced as follows.

Definition 10 (input safe atom). An atom is called input safe if (1) each of its input positions is filled in with a generic expression for this positions type, and (2) either the types of all input positions are ground or the terms filling in the input positions are mutually variable-disjoint.

In particular, an atom is input safe if the types of all input positions are ground. The notion of input safe atom is interesting for the following reason. The parameter passing mechanism in the unification of a simply moded query $A$, which is correctly typed in its input positions, with an input safe head atom $H$ is as follows: first the input values are passed from the atom $A$ to the head $H$, then the output values are passed from $H$ to $A$. The next proposition generalizes this informal argument (we refer to [15] for more details). In the sequel, we abbreviate simply moded well-typed with SMWT.

Proposition 1. Let $P$ be a SMWT program and $\leftarrow B_{1}, \ldots, B_{n}$ be a SMWT query. Suppose that the heads of the clauses of $P$ are input safe.

If $\sigma=\theta^{1} \ldots \theta^{k}$ is the substitution of a (partial) LD-derivation of $\leftarrow B_{1}, \ldots, B_{n}$ in $P$, then the input arguments of $B_{1}$ are equal to the input arguments of $B_{1} \sigma$ modulo variable renaming.

It follows from Proposition 1 that, if $P$ and $\leftarrow A$ are SMWT and if the heads of the clauses in $P$ are input safe, then in every LD-derivation of $P \cup\{\leftarrow A\}$ the input arguments of $A$ get never instantiated. We will use this in the termination condition for SMWT programs and queries (Theorem 2). Namely, if the heads of the clauses of the program are input safe, we are no longer forced to reason on "calls" in the termination condition, instead, the termination condition can be easily stated at the clause level, which is useful in the context of an automatic termination analysis.

In [2] it was shown that if a program $P$ and query $\leftarrow A$ are SMWT and if the heads of the clauses in $P$ are input safe, $P \cup\{\leftarrow A\}$ is unification free (i.e. unification can be replaced by iterated matching). We refer to the list of program examples in the discussion section of [2], where appropriate modings and typings for the considered predicates are given such that the programs are SMWT and the heads of their clauses are input safe. Note that for surprisingly many programs appropriate modings and typings can be found.

\subsection{Norms and Level Mappings}

We recall the definitions of norm and level mapping. 
Definition 11 (norm, level mapping). Let $P$ be a definite program. A norm is a function $\|\|:. U_{P}^{E} \rightarrow \mathrm{N}$. A level mapping is a function $||:. B_{P}^{E} \rightarrow \mathbb{N}$.

We introduce some notation. By Pred $_{P}$, Fun $_{P}$, Const $_{P}$ and $\operatorname{Var}_{P}$ we denote the sets of predicate, function, constant symbols and variables of $P$ respectively. The set of predicate coefficients, $P C(P)$, respectively functor coefficients, $F C(P)$, associated to a program $P$ are the sets of symbols $P C(P)=\left\{p_{i} \mid p / n \in\right.$ Pred $\left._{P} \wedge i \in\{1, \ldots, n\}\right\}$, and $F C(P)=\left\{f_{i} \mid f / m \in F u n_{P} \wedge i \in\{0,1, \ldots, m\}\right\}$. Here, all norms and level mappings will be of a specified form (for the norms this is a slight variant of the semi-linear norms [3]).

Definition 12 (symbolic norm and level mapping, symbol mapping). The symbolic norm $\|.\|^{S}$ and symbolic level mapping |. $\left.\right|^{S}$ are defined as:

$$
\begin{aligned}
& \left\{\begin{array}{l}
\|X\|^{S}=\|c\|^{S}=0 \\
\left\|f\left(t_{1}, \ldots, t_{m}\right)\right\|^{S}=f_{0}+\sum_{i=1}^{m} f_{i}\left\|t_{i}\right\|^{S}
\end{array} \text { for } X \in \operatorname{Var}_{P}, c \in \text { Const }_{P},\right. \\
& \left|p\left(t_{1}, \ldots, t_{n}\right)\right|^{S}=\sum_{i=1}^{n} p_{i}\left\|t_{i}\right\|^{S}
\end{aligned}
$$

with $f_{i} \in F C(P)$, for $i \in\{0, \ldots, m\}$ and $p_{i} \in P C(P)$, for $i \in\{1, \ldots, n\}$. $A$ symbol mapping is a mapping $s: F C(P) \cup P C(P) \rightarrow \mathbb{N}$.

A symbol mapping $s$ induces in a natural way a norm and a level mapping, by mapping the coefficient symbols in the symbolic norm and level mapping to their actual values under $s$. The norm, resp. level mapping, induced by the symbol mapping $s$ is denoted by $\|\cdot\|_{s}$, resp. $|\cdot|_{s}$. For example, the term-size norm is induced by the symbol mapping $s$ with $s\left(f_{i}\right)=1, i=0, \ldots, n$, for all $f / n \in F$ un $_{P}$.

Next, we define the notion of finitely partitioning level mapping. This notion is crucial in the context of a termination analysis for tabled logic programs. In particular, in the termination condition of [7], it is required that the level mapping is finitely partitioning on the call set of the program w.r.t. the set of queries for which one wants to prove termination (see also Theorem 1).

Definition 13 (finitely partitioning level mapping). Let $|$.$| be a level map-$ ping and $S \subseteq B$. The level mapping $|$.$| is called finitely partitioning on S$ iff $\forall n \in \mathbb{N}: \sharp\left((|.|)^{-1}(n) \cap S\right)<\infty$, with $\sharp$ the cardinality function.

It is easy to see that if the set $S$ is finite, all level mappings are finitely partitioning on $S$ (in particular this is the case if the Herbrand Universe is finite). Intuitively it is clear that, for infinite sets $S$ closed under substitutions, a level mapping $|.|_{s}$ is finitely partitioning on $S$ if it takes into account enough argument positions of predicates and functors in $S$ (more precisely, for every atom $A$ in $S$ and every variable $X$ in $A$, at least one occurrence of $X$ in $A$ is taken into account, i.e. $s$ is not 0 there). We refer to [15] for a formal statement.

In order to automatize termination proofs, and in particular, in order to have a sufficient condition for termination which is stated fully at the clause level (and 
not on "calls"), most approaches rely on the notion of rigid level mapping. In particular, in [8], it is required that the level mapping is rigid on the call set of the program w.r.t. the set of queries for which one wants to prove termination. A level mapping is rigid on a set of atoms, if the value of an atom of the set is invariant under substitutions. As was shown in [3] (see also [15]), a level mapping $|\cdot|_{s}$ is rigid on a set $S$ if it does not take into account too many argument positions of predicates and functors in $S$ (more precisely, for every atom $A$ in $S$ and every variable $X$ in $A$, no occurrence of $X$ in $A$ is taken into account, i.e. $s$ is 0 there).

As follows from the informal argumentation above, the rigidity condition on the level mapping, as it appears in the automated termination analysis of [8], is difficult to combine with the condition on the level mapping to be finitely partitioning, as it appears in the termination condition for tabled logic programs of [7]. In our search for automatizable termination proofs for tabled logic programs, we solve this problem by abandoning the rigidity requirement and using other syntactical conditions on the program and set of queries which allow us to formulate an easy to automatize termination condition which is stated fully at the clause level. In order to find such conditions we use mode and type information. The following concepts will be useful.

Definition 14 (measuring only/all input). Let $|.|_{s}$ be a level mapping (induced by the symbol mapping s) and $S \subseteq B_{P}^{E}$.

We say that $|.|_{s}$ measures only input positions in $S$ iff for every predicate $p / n$ occurring in $S$ : if for $i \in\{1, \ldots, n\} s\left(p_{i}\right) \neq 0$, then $m_{p}(i)=$ In.

We say that $|.|_{s}$ measures all input positions in $S$ iff (1) for every predicate $p / n$ occurring in $S$ : if $m_{p}(i)=I n$, then $s\left(p_{i}\right) \neq 0$, and (D) for every functor $f / m, m>0$, occurring in an input position of an atom in $S: s\left(f_{i}\right) \neq 0$ for all $i \in\{0, \ldots, m\}$.

In the case of simply moded programs and queries, a level mapping is finitely partitioning on the call set if it measures all input positions in the call set.

Proposition 2. Let $P$ be a simply moded program and $S$ be a set of simply moded queries. Let $|.|_{s}$ be a level mapping which measures all input positions in $\operatorname{Call}(P, S)$. Then, $|.|_{s}$ is finitely partitioning on $\operatorname{Call}(P, S)$.

The notion of a level mapping measuring only input positions in $\operatorname{Call}(P, S)$ will allow us to state the termination conditions fully at the clause level. Note that the conditions on a level mapping to measure all and only input positions in the call set, can be combined without problems.

\section{Quasi-Termination}

We recall the definition of quasi-termination [7].

Definition 15 (quasi-termination). Let $P$ be a program and $S \subseteq B_{P}^{E}$. $P$ quasi-terminates w.r.t. $S$ iff for all $A$ such that $\tilde{\tilde{A}} \in S$, the $\bar{L} G$-forest for $P \cup\{\leftarrow A\}$ consists of a finite number of LG-trees. 
Note that the program $N A T$ of Example 1 quasi-terminates w.r.t. $\{$ nat $(X)\}$. In [7, Lemma 3.1] the following equivalence was proven: $P$ quasi-terminates w.r.t. $S$ iff for every $A$ in $S, C$ all $(P,\{A\})$ is finite. It is easy to see that LD-termination of $P$ w.r.t. $S$ (i.e. termination of $P$ w.r.t. $S$ under LD-resolution) implies quasitermination of $P$ w.r.t. $S$. Note that when a program is quasi-terminating w.r.t. a query $Q$, there are a finite number of trees in the LG-forest, all of them have finite branches, but, possibly, they have infinitely branching nodes. The stronger notion of $L G$-termination takes this source of nontermination into account. Namely, a program $P$ is said to LG-terminate w.r.t. $S$ iff for all $A$ such that $\tilde{A} \in S$, the LG-forest for $P \cup\{\leftarrow A\}$ consists of a finite number of finite LG-trees. Note that the program $N A T$ of Example 1 does not LG-terminate w.r.t. $\{$ nat $(X)\}$. By the lack of space, we will not consider LG-termination in this paper. We refer to [15] instead.

In [7], the quasi-acceptability condition was introduced and it was shown to be equivalent with quasi-termination [7, Theorem 3.1].

Theorem 1 (quasi-acceptability w.r.t. $S$ ). Let $P$ be a program and $S \subset$ $B_{P}^{E} . P$ quasi-terminates w.r.t. $S$ iff there is a level mapping $|$.$| which is finitely$ partitioning on Call $(P, S)$ such that

- for every atom $A$ such that $\tilde{A} \in \operatorname{Call}(P, S)$,

- for every clause $H \leftarrow B_{1}, \ldots, B_{n}$ in $P$ such that $m g u(A, H)=\theta$ exists,

- for every $i \in\{1, \ldots, n\}$, and every LD-computed answer substitution $\theta_{i-1}$ for $\leftarrow\left(B_{1}, \ldots, B_{i-1}\right) \theta$,

$$
|A| \geq\left|B_{i} \theta \theta_{i-1}\right|
$$

The aim of this paper is to extend the automatic constraint-based termination analysis for LD-resolution of [8], to prove quasi-termination in an automatic way. In order to do so, we need (1) a termination condition which is stated fully at the clause level (and not on "calls" as in the quasi-acceptability condition of Theorem 1), and (2) a syntactical condition on a level mapping in order to be finitely partitioning on the call set. In the next Subsection 3.1, we present such a condition in the case of SMWT programs and queries. As we will point out in Subsection 3.2, this condition forms the basis for extending the automatic approach of [8], in order to prove quasi-termination of SMWT programs and queries in an automatic way.

\subsection{Simply Moded Well-Typed Programs and Queries}

Proposition 2 provides us with a syntactical condition on a level mapping to be finitely partitioning on the call set of a simply moded program w.r.t. a set of simply moded queries: the level mapping has to measure all input positions in the call set. In order to be able to state a condition for quasi-termination which reasons fully at the clause level, we use Proposition 1. In Proposition 1, we proved that for a SMWT program $P$ and query $\leftarrow A$, such that the heads of the clauses of $P$ are input safe, the input arguments of $A$ get never instantiated 
during an LD-derivation. This allows us to state a condition at the clause level, if we require in addition that the level mapping measures only input positions in the call set.

Theorem 2. Let $P$ be a $S M W T$ program and $S \subseteq B_{P}^{E}$ be a set of $S M W T$ queries. Suppose that the heads of the clauses in $P$ are input safe. If there is a model $M$ for $P$ and a level mapping $|.|_{s}$ which measures all and only the input positions in Call $(P, S)$, such that

- for every clause $H \leftarrow B_{1}, \ldots, B_{n}$ in $P$,

- for $i \in\{1, \ldots, n\}$ and every substitution $\psi$ such that $M \models\left(B_{1} \wedge \ldots \wedge B_{i-1}\right) \psi$ and $(H \psi)^{I n},\left(B_{1} \psi\right)^{I n}, \ldots,\left(B_{i} \psi\right)^{I n} \in \operatorname{Call}(P, S)^{I n}$,

$$
|H \psi|_{s} \geq\left|B_{i} \psi\right|_{s},
$$

then $P$ is quasi-acceptable w.r.t. S. Hence, P quasi-terminates w.r.t. S.

Note that, because $P$ and $S$ are well-typed, it follows that, if the conditions of the theorem are satisfied, i.e. if $\psi$ is a substitution such that $M \models$ $\left(B_{1} \wedge \ldots \wedge B_{i-1}\right) \psi$ and $(H \psi)^{I n},\left(B_{1} \psi\right)^{I n}, \ldots,\left(B_{i} \psi\right)^{I n} \in \operatorname{Call}(P, S)^{I n}$, then $H \psi$ is correctly typed in its input positions, $\left(B_{1}, \ldots, B_{i-1}\right) \psi$ is correctly typed and $B_{i} \psi$ is correctly typed in its input positions.

We want to note that Theorem 2 can also be applied in the case of simply moded well-moded programs and queries. We already noted in Subsection 2.2 that the notion of well-modedness [1] is a special case of the notion of well-typedness: namely, well-typedness collapses to well-modedness if the types of all predicate positions are defined as Ground. Recall also from Subsection 2.2 that all terms are generic expressions for the Ground type, so the heads of all clauses of a well-moded program (in which we defined the types of all predicate positions as Ground) are trivially input safe. Finally, note that in the case of a well-moded program and set of queries, the condition on the level mapping to measure only input positions in the call set, has as a consequence that the level mapping is rigid on the call set. We refer to [15] for more details.

Example 2. Let $P$ be the following program computing the cyclic permutations of a list.

$$
\begin{array}{ll}
\operatorname{splitlast}([X],[], X) & \leftarrow \\
\operatorname{splitlast}([X \mid L],[X \mid R], Y) & \leftarrow \operatorname{splitlast}(L, R, Y) \\
\text { cycperm }(L, L) & \leftarrow \\
\text { cycperm }(L, R) & \leftarrow \operatorname{splitlast}(L, T, X), \operatorname{cycperm}([X \mid T], R)
\end{array}
$$

with splitlast $(<$ In : List $\rangle,\langle$ Out : List $\rangle,\langle$ Out $: U\rangle)$ and cycperm $(<$ In : List $>,<$ Out : List $>)$. Let $S$ be the set of queries $\{\operatorname{cycper} m(l, X) \mid l \in$ List $\}$. We prove that $P$ quasi-terminates w.r.t. $S$ by applying Theorem 2 (note that $P$ even LG-terminates w.r.t. $S$, a proof can be found in [15]; note however that $P$ does not LD-terminate w.r.t. $S$ ). Let's abbreviate splitlast to $s p$ and cycperm to cyc. 
$P$ and $S$ are well-typed. This is trivial to see for the set $S$. The program $P$ is well-typed because the following type judgements are true:

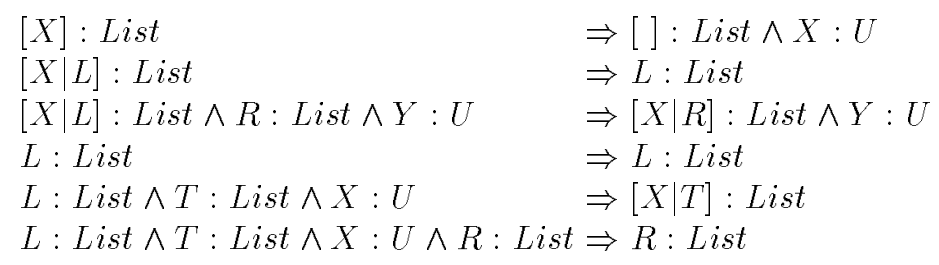

Also, $P$ and $S$ are simply moded and the heads of the clauses in $P$ are input safe. Let $|.|_{s}$ be the following level mapping (measuring all and only the input positions in $\operatorname{Call}(P, S))$ :

$$
\begin{aligned}
& \left|s p\left(t_{1}, t_{2}, t_{3}\right)\right|_{s}=\left\|t_{1}\right\|_{s}, \\
& \left|c y c\left(t_{1}, t_{2}\right)\right|_{s}=\left\|t_{1}\right\|_{s},
\end{aligned}
$$

with $\|.\|_{s}$ the term-size norm.

Consider the recursive clause for splitlast. For every $\psi,|s p([X \mid L],[X \mid R], Y) \psi|_{s}=$ $\|[X \mid L] \psi\|_{s}=1+\|L \psi\|_{s} \geq\|L \psi\|_{s}=|\operatorname{sp}(L, R, Y) \psi|_{s}$. Consider the recursive clause for cycperm. Again, for every $\psi,|\operatorname{cyc}(L, R) \psi|_{s}=\|L \psi\|_{s} \geq\|L \psi\|_{s}=$ $|s p(L, T, X) \psi|_{s}$. Let $\psi$ be a substitution such that $\operatorname{sp}(L, T, X) \psi$ is a consequence of the program and such that $c y c(L, R) \psi, \operatorname{sp}(L, T, X) \psi$ and $c y c([X \mid T], R) \psi$ are input-correct w.r.t. $\operatorname{Call}(P, S)$. Note that then, $\|L \psi\|_{s} \geq 1+\|X \psi\|_{s}$ $+\|T \psi\|_{s}=\|[X \mid T] \psi\|_{s}$. Thus, $|\operatorname{cyc}(L, R) \psi|_{s}=\|L \psi\|_{s} \geq\|[X \mid T] \psi\|_{s}=$ $|\operatorname{cyc}([X \mid T], R) \psi|_{s}$. Hence, by Theorem 2, $P$ quasi-terminates w.r.t. $S$.

\subsection{Constraint-Based Approach for Automatically Proving Quasi-Termination}

In this subsection, we point out how the constraint-based approach of [8] for automatically proving LD-termination of definite programs w.r.t. sets of queries needs to be changed in order to prove quasi-termination in an automatic way. We restrict ourselves to SMWT programs and queries such that the heads of the program clauses are input safe. The basis of the automatic approach towards quasi-termination is Theorem 2 of the previous subsection.

We first recall the main ideas of [8]. In [8], a new strategy for automatically proving LD-termination of logic programs w.r.t. sets of queries is developed. A symbolic termination condition is introduced, called rigid acceptability, by parametrising the concepts of norm, level mapping and model. In order to symbolize the notion of model, symbolic versions of interargument relations are introduced. Interargument relations are abstractions of interpretations by specifying relations which hold between the norms of certain arguments of their member atoms. In [8], interargument relations express an inequality relation. The rigid acceptability condition is translated into a system of constraints on the values of the introduced symbols only. A system of constraints identifies sets of suitable norms, level mappings and interarguments relations which can be used in the 
termination condition. In other words, if a solution for the constraint system exists, termination can be proved. The solving of constraint sets enables the different components of a termination proof to communicate with one another and to direct the proof towards success (if there is). The method of [8] is both efficient and precise.

We adapt the approach of [8] to prove quasi-termination of SMWT programs w.r.t. sets of SMWT queries, such that the clauses of the programs have input safe heads, by using Theorem 2. Note that this theorem is stated fully at the clause level. As can be seen from Theorem 2, quasi-termination is implied by the following conditions on the introduced symbols for norm, level mapping and interargument relations.

(i) The level mapping has to measure all and only input positions in $\operatorname{Call}(P, S)$.

(ii) Every introduced interargument relation must be valid (meaning that the induced interpretation is a model of $P$ ).

(iii) For every clause $H \leftarrow B_{1}, \ldots, B_{n}$ in $P$, for $i \in\{1, \ldots, n\}$, and for every substitution $\psi$ such that $B_{1} \psi, \ldots, B_{i-1} \psi$ belong to their valid interargument relations and such that $(H \psi)^{I n},\left(B_{1} \psi\right)^{I n}, \ldots,\left(B_{i} \psi\right)^{I n} \in \operatorname{Call}(P, S)^{I n}$, the weak inequality $|H \psi| \geq\left|B_{i} \psi\right|$ must hold.

Except maybe for condition (i), the modifications needed to transform the constraints of [8] into the constraints for quasi-termination, are straightforward, and we will not elaborate on them (we refer to [15] and [8]). We show in the following example how the first condition $(i)$ is translated into symbolic constraints.

Example 3. Recall the SMWT program $P$ and set $S$ of Example 2. Note that $\operatorname{Call}(P, S)=\{c y c(l, X), s p(l, R, Y) \mid l \in$ List $\}$. We introduce symbolic versions for the norm and level mapping (see Definition 12). Let $t \in U_{P}^{E}$, then the symbolic norm on $t$ is defined as:

$$
\begin{cases}\|X\|^{S}=\|[]\|^{S}=0 & \text { for } X \in \operatorname{Var}_{P},[] \in \text { Const }_{P} \\ \|t\|^{S}=[. \mid \cdot]_{0}+[. \mid \cdot]_{1}\left\|t_{1}\right\|^{S}+[. \mid \cdot]_{2}\left\|t_{2}\right\|^{S} & \text { for } t=\left[t_{1} \mid t_{2}\right],\end{cases}
$$

with $\left\{[. \mid .]_{0},[. \mid .]_{1},[. \mid]_{2}\right\}=F C(P)$. Let $s p\left(t_{1}, t_{2}, t_{3}\right), c y c\left(t_{1}, t_{2}\right) \in B_{P}^{E}$, then the symbolic level mapping is defined as:

$$
\begin{aligned}
& \left|s p\left(t_{1}, t_{2}, t_{3}\right)\right|^{S}=s p_{1}\left\|t_{1}\right\|^{S}+s p_{2}\left\|t_{2}\right\|^{S}+s p_{3}\left\|t_{3}\right\|^{S}, \\
& \left|c y c\left(t_{1}, t_{2}\right)\right|^{S}=c y c_{1}\left\|t_{1}\right\|^{S}+c y c_{2}\left\|t_{2}\right\|^{S}
\end{aligned}
$$

with $\left\{s p_{1}, s p_{2}, s p_{3}, c y c_{1}, c y c_{2}\right\}=P C(P)$.

The condition on the level mapping to measure only input positions in $\operatorname{Call}(P, S)$ is expressed in the following constraints: $s\left(c y c_{2}\right)=0, s\left(s p_{2}\right)=0$ and $s\left(s p_{3}\right)=0$. Condition $(i)$ also requires that the level mapping measures all input positions. This gives rise to the following constraints: $s\left(c y c_{1}\right) \neq 0, s\left(s p_{1}\right) \neq 0, s\left([. \mid \cdot]_{0}\right) \neq 0$, $s\left([. \mid \cdot]_{1}\right) \neq 0$ and $s\left([. \mid \cdot]_{2}\right) \neq 0$.

Note that the level mapping proposed in Example 2 is a solution for the set of 8 constraints above and indeed, that level mapping measures all and only input positions in $\operatorname{Call}(P, S)$. 


\section{Conclusions and Related Works}

In this paper, we investigated the problem of automatically proving termination of logic programs under a tabled execution mechanism. Two works form the basis of this paper: [7], where the notion of quasi-termination is introduced and a necessary and sufficient condition (quasi-acceptability) is given, and [8], where an automatic approach towards LD-termination is developed. It turned out that the rigidity condition on the level mapping, as it appears in the automated termination analysis of [8], is difficult to combine with the condition on the level mapping to be finitely partitioning, as it appears in the quasi-acceptability condition of [7]. In this paper, we showed that for simply moded well-typed programs such that the heads of the clauses are input safe, a sufficient condition for quasi-termination can be given, which is formulated fully at the clause level and which is easy to automatize. We pointed out how this sufficient condition can be automatized by extending the approach of [8]. Due to space limitations, we did not include our results on the stronger notion of LG-termination. We refer to [15] instead.

Since all programs that terminate under LD-resolution, are quasi-terminating and LG-terminating as well, verification of termination under LD-resolution using an existing automated termination analysis (such as those surveyed in e.g. [6]) is a sufficient proof of the programs quasi-termination and LG-termination. In the recent paper [9], Etalle et al study how mode information can be used for characterizing properties of LD-termination. They define and study the class of well-terminating programs, i.e. programs for which all well-moded queries have finite LD-derivations. They introduce the notion of well-acceptability and show that for well-moded programs, well-acceptability implies well-termination.

Termination proofs for (S)LD-resolution are sufficient to prove termination under a tabled execution mechanism, but, since there are quasi-terminating and LG-terminating programs, which are not LD-terminating, more effective proof techniques can be found. There are only relatively few works studying termination under a tabled execution mechanism. We already discussed the work of [7], which forms the basis of this paper. In [13], in the context of wellmoded programs, a sufficient condition is given for the bounded term-size property, which implies LG-termination. [10] provides another sufficient condition for quasi-termination in the context of functional programming. In parallel with the work reported on in this paper, in [16] the authors of this paper together with K. Sagonas, investigated an orthogonal extension of the work of [7]. Namely, in [16], termination under a mix of tabled and Prolog execution is considered and, besides a characterisation of the two notions of universal termination under such a mixed execution, modular termination conditions are given. An integration of [16] with the results of this paper is straightforward.

We plan to implement the constraint-based technique for automatically proving quasi-termination and LG-termination (note that a prototype implementation for automatically proving LD-termination [8] exists and is available at our site). Also, it remains to be studied how our results can be extended to automatically prove quasi-termination and LG-termination for a larger class of 
programs and queries (i.e. for programs and queries which are not simply moded well-typed). Finally, the study of termination of normal logic programs under tabled execution mechanism is an interesting topic for future research.

\section{References}

1. K.R. Apt and E. Marchiori. Reasoning about Prolog programs: from modes through types to assertions. Formal Aspects of Computing, 6(6A):743-765, 1994.

2. K.R. Apt and S. Etalle. On the unification free Prolog programs. In A. Borzyszkowski and S. Sokolowski, editors, Proc. MFCS, pages 1-19. number 771 in LNCS, Springer-Verlag, 1993.

3. A. Bossi, N. Cocco, and M. Fabris. Norms on terms and their use in proving universal termination of a logic program. Theoretical Computer Science, 124(2):297-328, 1994.

4. F. Bronsard, T. Lakshman, and U. Reddy. A framework of directionality for proving termination of logic programs. In K.R. Apt, editor, Proc. JICSLP, pages 321-335. MIT Press, 1992.

5. W. Chen and D. S. Warren. Tabled Evaluation with Delaying for General Logic Programs. J. ACM, 43(1):20-74, 1996.

6. D. De Schreye and S. Decorte. Termination of logic programs: the never-ending story. Journal of Logic Programming, 19 \& 20:199-260, 1994.

7. S. Decorte, D. De Schreye, M. Leuschel, B. Martens, and K. Sagonas. Termination Analysis for Tabled Logic Programming. In N. Fuchs, editor, Proc. LOPSTR, number 1463 in LNCS, pages 107-123. Springer-Verlag, 1997.

8. S. Decorte, D. De Schreye, and H. Vandecasteele. Constraint-based automatic termination analysis for logic programs. ACM TOPLAS. to appear.

9. S. Etalle, A. Bossi, and N. Cocco. Termination of well-moded programs. Journal of Logic Programming, 38(2):243-257, 1998.

10. C. K. Holst. Finiteness Analysis. In J. Hughes, editor, Proc. of the 5th ACM Conference on FPCA, number 523 in LNCS, pages 473-495. Springer-Verlag, 1991.

11. T. Kanamori and T. Kawamura. OLDT-based abstract interpretation. Journal of Logic Programming, 15(1 \& 2):1-30, 1993.

12. J. Lloyd. Foundations of logic programming. Springer-Verlag, 1987.

13. L. Plümer. Termination proofs for logic programs. Number 446 in LNAI. SpringerVerlag, 1990

14. H. Tamaki and T. Sato. OLD Resolution with Tabulation. In Proc. ICLP, number 225 in LNCS, pages 84-98. Springer Verlag, 1986.

15. S. Verbaeten and D. De Schreye. Termination analysis of tabled logic programs using mode and type information. Technical Report 277, Department of Computer Science, K.U.Leuven. Available at http://www.cs.kuleuven.ac.be/ sofie.

16. S. Verbaeten, K. Sagonas, and D. De Schreye. Modular termination proofs for Prolog with tabling. Technical report, Department of Computer Science, K.U.Leuven, 1999. Available at http://www.cs.kuleuven.ac.be/ sofie (accepted for PPDP'99). 